\title{
Differential Rotation as an Axisymmetric Resonant Mode of Convection
}

\author{
Kwing L. Chan ${ }^{1}$, Hans G. Mayr ${ }^{2}$ \\ ${ }^{1}$ Applied Research Corporation, Landover, MD, USA \\ ${ }^{2}$ Goddard Space Flight Center, Greenbelt, MD, USA
}

Recent results from helioseismology (see Goode, these Proceedings) have shown that the inferred contours of the solar angular velocity are more or less radial in the convection region, and the rotation becomes uniform below. These observations contradict the prevailing numerical models of Taylor columns which predict angular velocity contours parallel to the rotation axis of the Sun. Thus, an alternative explanation of solar differential rotation is called for.

Presently, it is not feasible to construct a thermally-relaxed, dynamically selfconsistent numerical model of the solar convection zone (see Chan and Serizawa, these Proceedings). It is then appropriate to explore simplified models that may shed some light. A number of analytical models have been proposed for the solar differential rotation, and the reader is referred to the book by Rüdiger (1989) for a comprehensive review of this subject. Here, we report on some recent development on the convective resonance model proposed by Chan et al. (1987; hereafter referred as CSM).

In the convective resonance model, differential rotation is described in terms of the zonally symmetric velocity field. The model is linear, axisymmetric, and eddy diffusion describes the Reynolds stresses that dissipate energy and momentum. Latitudinal variations of convective heat transfer (Weiss, 1965; Durney and Roxburgh, 1971; Belvedere and Paternò, 1977) are invoked to drive the motions. An important aspect of the model is that the superadiabatic gradient is dynamically important; and a resonant convective eigenmode then develops which is rather insensitive to the magnitude and latitude dependence of the excitation source.

Using scale analysis, we illustrate how such a resonance develops. With standard notations, the linearized conservation equations, for the zonally averaged temperature perturbation, $\delta T$, meridional velocity, $V$, and zonal velocity, $U$, are written in the simplified form

$$
\begin{gathered}
\frac{\Delta T}{\operatorname{Pr} \tau_{d}}-\frac{T \delta \nabla}{\lambda H} V=q, \\
\frac{g d}{\lambda T} \Delta T-\Omega U-\frac{V}{\tau_{d}}=0,
\end{gathered}
$$




$$
\Omega V-\frac{U}{\tau_{d}}=0,
$$

where $q$ is the excitation source, $\Omega$ the mean rate of rotation, $\lambda$ and $d$ the horizontal and vertical length scales, $H$ the pressure scale height, $\tau_{d}=d^{2} / \nu$ the dissipation time constant, and $\operatorname{Pr}$ the Prandtl number; the hydrostatic approximation $\Delta \ln p=$ $\Delta T d / T H$ is used. The solution then is

$$
\begin{gathered}
V=\frac{\operatorname{Pr} g d}{T \lambda\left[\Omega^{2}+\tau_{d}^{-2}-\frac{g \operatorname{Pr} \delta \nabla d^{2}}{\lambda^{2} H}\right]} q, \\
U=\Omega \tau_{d} V=\sqrt{\mathrm{Ta}} V .
\end{gathered}
$$

Since the Taylor number is large, Ta $\gg 1$, and the solar envelope is convectively unstable, i.e. $\delta \nabla>0$, the denominator in Eq. (4) can vanish. A resonance then develops such that the wavelength

$$
\lambda=\frac{d}{\Omega} \sqrt{\frac{g \operatorname{Pr} \delta \nabla}{H}}
$$

is preferentially excited. When the conservation equations are formulated for a spherical envelope, using spherical harmonics, only discrete wavelengths can be excited.

For the simplified one-layer Boussinesq model of CSM it was shown that the fundamental resonance mode matches the surface differential rotation of the sun. In the following, we present a compressible model which accounts for stratification; the resulting differential rotation in the solar interior is discussed and compared with observations.

Our stratified model is spectral in terms of zonal spherical harmonics and is constructed with the following specifications: (i) The linearized hydrodynamic equations are solved for the response to a small source (described with the spherical harmonic, $P_{2}$ ) in the energy equation. (ii) The domain of computation extends from 0.57 to 0.93 solar radii, and the bottom of the convection zone is at 0.69 . (iii) The distribution of the superadiabatic gradient $\delta \nabla$ in the convection zone is determined by $\nu \rho C_{p} T \delta \nabla / \operatorname{Pr} H=$ flux; where $\nu$, the diffusivity is constant, and $\operatorname{Pr}=0.3 ; \rho$ is the density; $C_{p}$ is the specific heat under constant pressure; i.e. $\delta \nabla \propto \rho^{-1}$. (iv) The stratification of the stable layer below the convection zone is based on a standard solar model. (v) No-slip boundary conditions are adopted at the bottom and stress-free conditions at the top; (vi) The degree of spherical harmonics is up to 6 . (vii) The number of vertical grid points is 100 .

A search for resonance was performed by varying $\delta \nabla_{0}$, the value of the superadiabatic gradient at the top of the convection layer. The lowest order resonance occurs at $\delta \nabla_{0}=3.7 \times 10^{-4}$ (with $\delta \nabla \sim 5 \times 10^{-5}$ in the middle of the convective layer, $\nu=1.2 \times 10^{10} \mathrm{~cm}^{2} \mathrm{~s}^{-1}$ ).

We show the computed angular velocity distribution as a surface plot in Fig. 1 and the observations (Dziembowski et al., 1989) in Fig. 2. Latitude and depth are represented by horizontal coordinates, and the angular velocity by the vertical 


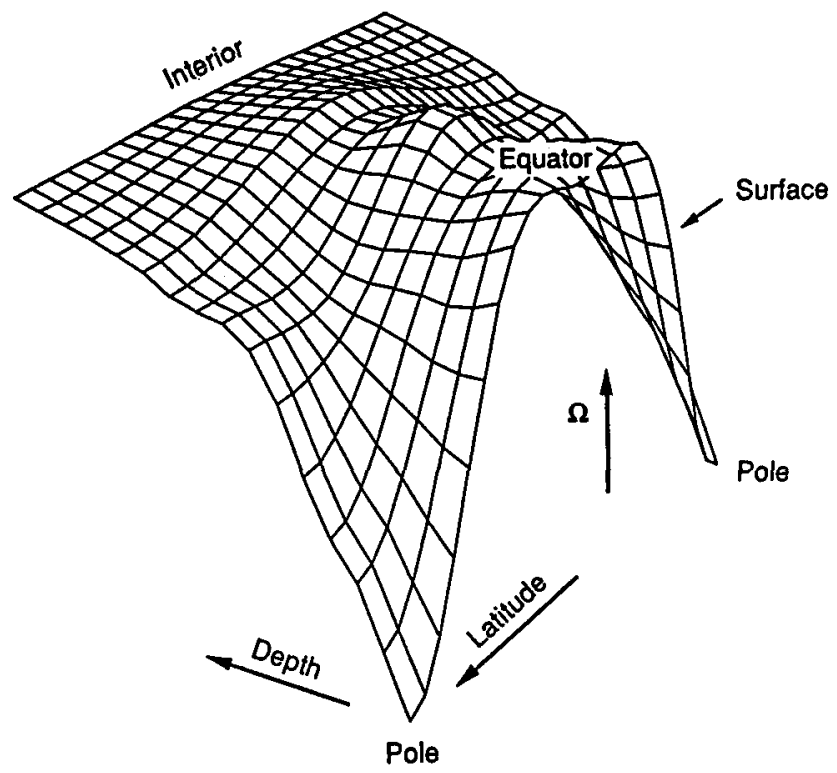

Fig. 1. Computed differential rotation

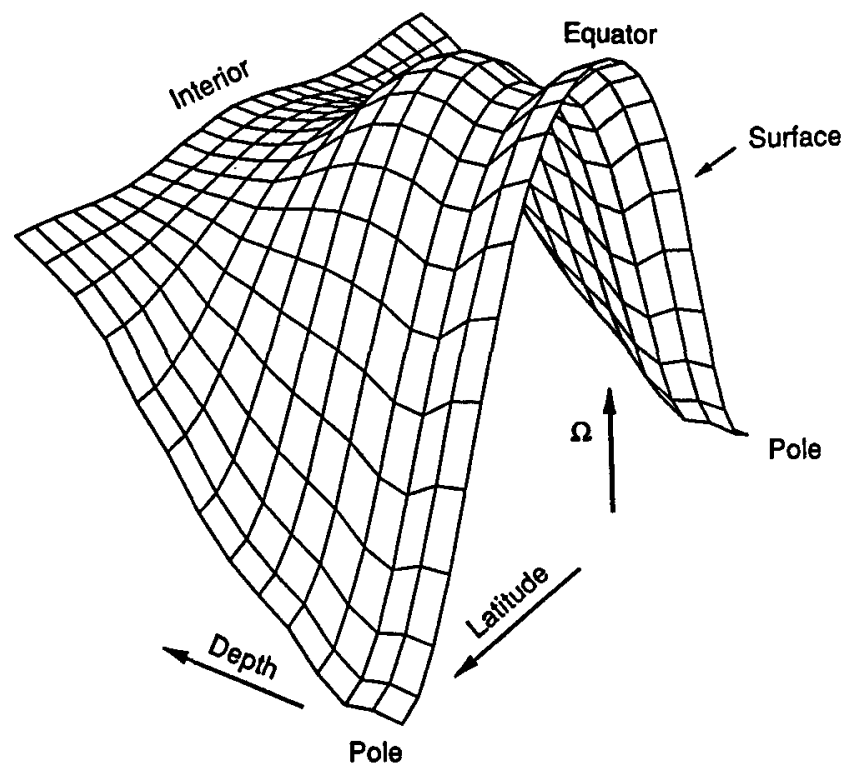

Fig. 2. Observed solar differential rotation (Dziembowski et al., 1989) 
coordinate. The velocity distribution is very similar to that obtained from helioseismology, and when its magnitude is matched to that observed at the surface, the maximum meridional velocity is only about $10 \mathrm{~cm} / \mathrm{sec}$. There are also some disagreements. The small extraneous dip in the surface angular velocity at the equator is not observed, and the magnitude of the differential rotation decreases somewhat faster towards the bottom of the convection zone. However, these discrepancies are not serious, considering that the model has been constructed with a simple set of assumptions without any fine tuning. The model needs to be explored further by varying the distributions of $\delta \nabla$ and $\nu$ in the convection zone.

Acknowledgement: KLC thanks NSF for support (AST-8815457)

\section{References}

Belvedere, G., Paternò, L.: 1977, Solar Phys. 54, 289

Chan, K.L., Sofia, S., Mayr, H.G.: 1987, In The internal solar angular velocity, eds. B.R. Durney and S. Sofia, Reidel, Dordrecht, p.347

Durney, B.R., Roxburgh, I.W.: 1971, Solar Phys. 16, 3

Dziembowski, W.A., Goode, P.R., Libbrecht, K.G.: 1989, Astrophys. J. 336, 53

Rüdiger, G.: 1989, Differential rotation and stellar convection: Sun and solar-type stars, Gordon and Breach, New York

Weiss, N.O.: 1965, Observatory 85, 37 